\title{
Isoliquiritigenin inhibits proliferation and metastasis of MKN28 gastric cancer cells by suppressing the PI3K/AKT/mTOR signaling pathway
}

\author{
XIU-RONG ZHANG ${ }^{1,2}$, SHI-YAO WANG ${ }^{3}$, WEN SUN $^{4}$ and $\mathrm{CHAO} \mathrm{WEI}^{5}$ \\ ${ }^{1}$ Department of Traditional Chinese Medicine, Maternal and Child Health Care of Shandong Province; \\ ${ }^{2}$ Key Laboratory of Birth Regulation of National Health and Family Planning Commission of China, Jinan, Shandong 250014; \\ ${ }^{3}$ Department of Chinese Medicine, The Second Clinical Medical College of Guangzhou University of Traditional Chinese Medicine, \\ Guangzhou, Guangdong 510000; ${ }^{4}$ Department of Research, Beijing Splinger Medical Research Institute, Jinan, \\ Shandong 250021; 5 Department of Ophthalmology, The Second Hospital of Shandong University, \\ Jinan, Shandong 250033, P.R. China
}

Received November 3, 2017; Accepted June 19, 2018

DOI: $10.3892 / \mathrm{mmr} .2018 .9318$

\begin{abstract}
Isoliquiritigenin (ISL) is a flavonoid extracted from licorice root, which is known to serve important antitumor roles in numerous types of cancers; however, its effect on gastric cancer remains to be elucidated. The present study aimed to explore the roles and underlying mechanisms of ISL in MKN28 gastric cancer cells. MKN28 cell proliferation was measured using the Cell Counting Kit-8 (CCK8) assay. A Transwell assay was used to determine the effects of ISL on the migration and invasion of MKN28 cells. Apoptosis was assessed by flow cytometry, and the expression levels of apoptosis-, autophagy- and signaling pathway-related proteins were detected by western blot analysis. The results of the CCK8 assay demonstrated that ISL significantly inhibited the proliferation of MKN28 cells $(\mathrm{P}<0.05)$. Transwell assays demonstrated that the migration and invasion of MKN28 cells were significantly inhibited following treatment with ISL $(\mathrm{P}<0.05)$. Flow cytometric analysis indicated that ISL induced apoptosis of MKN28 cells. In addition, western blot analysis revealed that the ratio of microtubule-associated proteins 1A/1B light chain 3B (LC3)II/LC3I was upregulated, as was Beclin 1 expression; however, p62 was downregulated
\end{abstract}

Correspondence to: Dr Wen Sun, Department of Research, Beijing Splinger Medical Research Institute, 685 Jingsi Road, Huaiyin, Jinan, Shandong 250021, P.R. China

E-mail: ormedu@163.com

Dr Chao Wei, Department of Ophthalmology, The Second Hospital of Shandong University, 247 Beiyuan Road, Jinan, Shandong 250033 , P.R. China

E-mail: doctorweichao@163.com

Key words: isoliquiritigenin, MKN28 cells, proliferation, phosphoinositide 3-kinase/protein kinase B/mammalian target of rapamycin, apoptosis, autophagy following ISL pretreatment, thus suggesting that ISL triggered autophagy in MKN28 cells. In addition, the phosphorylation levels of protein kinase B (AKT) and mammalian target of rapamycin (mTOR) were significantly reduced following ISL treatment. These results indicated that ISL may influence apoptosis and autophagy in MKN28 cells by suppressing the phosphoinositide 3-kinase/AKT/mTOR signaling pathway. In conclusion, the findings of the present study suggested that ISL may inhibit MKN28 cell proliferation, migration and invasion by inducing apoptosis and autophagy, implying potential as a therapeutic agent for gastric cancer.

\section{Introduction}

Gastric cancer is the fourth most prevalent cancer worldwide $(1,2)$ and the second leading cause of cancer-associated mortality (3). Despite the important advances in cancer therapy, gastric cancer remains a major malignancy and a serious threat to human health (4). Surgical, radiotherapeutic and chemotherapeutic strategies have recently become available for the treatment of gastric cancer (5); however, radiotherapy and chemotherapy exert severe adverse effects, and the development of drug resistance is common. Therefore, it is necessary to develop novel, effective and safe agents for the treatment of gastric cancer. Recently, natural products have attracted attention. Studies have reported that compounds from natural resources are suitable alternatives for controlling cancer with minimal toxicity and high efficacy (6-8).

Isoliquiritigenin (ISL) is a flavonoid with a chalcone structure that is derived from licorice compounds $(9,10)$. It is found ubiquitously in foods and beverages, and tobacco. ISL has been reported to exhibit a wide range of distinct biological properties and pharmacological activities, including anti-inflammatory, anti-oxidation, antiplatelet aggregation, cardioprotective effects against ischemia-reperfusion, and estrogenic properties (11-13). ISL has also been reported to suppress the proliferation and to induce the apoptosis of 
numerous cancers in vitro and in vivo (14), including colon and breast cancer (15-18).

At present, the anticancer effects and underlying mechanisms of ISL on gastric cancer have not been fully elucidated. The present study conducted a series of preliminary experiments to suggest that ISL may inhibit the proliferation, migration and invasion of MKN28 gastric cancer cells, which may be associated with phosphoinositide 3-kinase (PI3K)/protein kinase B $(\mathrm{AKT}) / \mathrm{mammalian}$ target of rapamycin (mTOR) signaling pathway-mediated apoptosis and autophagy.

\section{Materials and methods}

Cell culture and treatment. The MKN28 human gastric cancer cell line and the GES-1 human gastric normal epithelial mucosa cell line were purchased from the Cell Bank of the Chinese Academy of Sciences (Shanghai, China). The cells were cultured in RPMI-1640 medium supplemented with $10 \%$ (v/v) fetal bovine serum (Gibco; Thermo Fisher Scientific, Inc., Waltham, MA, USA), penicillin (100 U/ml) and streptomycin $(0.1 \mathrm{mg} / \mathrm{ml}$; both Sigma-Aldrich; Merck KGaA, Darmstadt, Germany) and incubated at $37^{\circ} \mathrm{C}$ in a humidified atmosphere containing $5 \% \mathrm{CO}_{2}$. When the cells entered the logarithmic growth phase, they were washed with PBS three times, and were then digested by trypsin (Beijing Solarbio Science \& Technology Co., Ltd., Beijing, China). Once the cells became rounded, the digestion was terminated and the cells were placed in culture medium, after which they were pipetted into 6 -well plates for subsequent experiments. When cell confluence in the wells reached $\sim 80 \%$, the cells were treated with ISL (MedChemExpress, Monmouth Junction, NJ, USA) or $0.1 \%$ dimethyl sulfoxide (DMSO; Amresco, LLC, Solon, $\mathrm{OH}$, USA) as a negative control (NC).

Cell Counting Kit-8 (CCK8) proliferation detection. CCK-8 (Beijing Solarbio Science \& Technology Co., Ltd. Beijing, China) was used to assess the effects of ISL on MKN28 and GES-1 cell proliferation. MKN28 or GES-1 cells $(100 \mu \mathrm{l})$ were seeded into 96-well plates at a cellular density of 1,000 cells/well. To the NC group, $0.1 \%$ DMSO was added, whereas $20 \mu \mathrm{M}$ ISL was added to the experimental group. Cells were cultured for $0,24,48$ and $72 \mathrm{~h}$ time intervals at $37^{\circ} \mathrm{C}$, and the cell viabilities at each time interval were subsequently detected. Prior to detection, $10 \mu \mathrm{l}$ CCK8 solution was added to each well for $1.5 \mathrm{~h}$ at $37^{\circ} \mathrm{C}$. The optical density (OD) value was measured at $450 \mathrm{~nm}$ using a microplate reader to obtain the proliferation curve.

Transwell migration and invasion assays. For the invasion assay, the inner layer of a 24-well Transwell system (EMD Millipore, Billerica, MA, USA) was pre-coated with Matrigel (BD Biosciences, Franklin Lakes, NJ, USA) at a 1:6 dilution. Cells treated with $20 \mu \mathrm{M}$ ISL or $0.1 \%$ DMSO for $24 \mathrm{~h}$ were suspended in serum-free medium and $100-\mu 1$ cell suspension $\left(\sim 1 \times 10^{4}\right.$ cells) was added to the upper chambers. The lower chambers were filled with culture medium. Following overnight incubation, cells on the surface of the upper chamber were removed with a cotton swab. Cells on the bottom were fixed in $4 \%$ paraformaldehyde for $0.5 \mathrm{~h}$ at room temperature, stained with $0.1 \%$ crystal violet dye for $20 \mathrm{~min}$ at room temperature and washed with PBS at room temperature. Subsequently, images were captured and the cells in five random fields were counted manually using light microscopy.

The migration assay was similar to the invasion assay, with the exception that the Transwell system was not coated with Matrigel, and the number of cells tested was 5,000.

Apoptosis analysis. Following treatment with $20 \mu \mathrm{M}$ ISL or $0.1 \%$ DMSO (control group) for $24 \mathrm{~h}$, cells were collected and digested with trypsin (Beijing Solarbio Science \& Technology Co., Ltd.). Cells were then washed using precooled PBS $\left(4^{\circ} \mathrm{C}\right)$. The cell density was adjusted to $1-5 \times 10^{6} / \mathrm{ml}$ by adding $1 \mathrm{X}$ binding buffer. Subsequently, 100- $\mu 1$ cell suspension was placed in a $5 \mathrm{ml}$ flow tube, and $5 \mu \mathrm{l}$ Annexin V/fluorescein isothiocyanate (FITC; BD Biosciences, Franklin Lakes, NJ, USA) was added and mixed by flicking the tube for $5 \mathrm{~min}$ in the dark at room temperature, followed by incubation with $10 \mu \mathrm{l}$ propidium iodide for 10-15 min in the dark room at room temperature. Following this, $400 \mu \mathrm{l}$ PBS was added and mixed, and the tubes were subsequently placed on ice. The results were detected using a flow cytometer (BD Biosciences) in $1 \mathrm{~h}$ and analyzed using FlowJo v10.0 software (FlowJo LLC, Ashland, OR, USA).

Western blot analysis. To extract proteins, cells in the experimental and NC groups were added to 6-well plates and treated with radioimmunoprecipitation assay lysis buffer (CW Biotech, Beijing, China) plus protease inhibitor. Protein concentration was measured using a Bicinchoninic Acid Protein Assay kit (CW Biotech), after which the proteins were heated for $5 \mathrm{~min}$ at $95^{\circ} \mathrm{C}$. Vertical electrophoresis was performed with $\sim 20 \mu \mathrm{g}$ protein in each lane. The proteins were isolated by $10 \%$ SDS-PAGE and transferred onto polyvinylidene fluoride membranes. The membranes were blocked with 5\% skim milk powder at room temperature for $1 \mathrm{~h}$ and were incubated with primary antibodies overnight at $4^{\circ} \mathrm{C}$. The following rabbit anti-human primary antibodies were used: AKT (1:1,000; cat. no. 9272; Cell Signaling Technology, Inc., Danvers, MA, USA), phosphorylated (p-)AKT (1:1,000; cat. no. 4060; Cell Signaling Technology, Inc.), mTOR (1:1,000; cat. no. 2972; Cell Signaling Technology, Inc.), p-mTOR (1:1,000; cat. no. 2971; Cell Signaling Technology, Inc.), B-cell lymphoma 2 (Bcl-2; 1:1,000; cat. no. 4223; Cell Signaling Technology, Inc.), caspase-3 (1:1,000; cat. no. 9664; Cell Signaling Technology, Inc.), Bcl-2-associated X protein (Bax; 1:1,000; cat. no. 5023; Cell Signaling Technology, Inc.), microtubule-associated proteins $1 \mathrm{~A} / 1 \mathrm{~B}$ light chain $3 \mathrm{~B}$ (LC3; 1:1,000; cat. no. 14600-1-AP; ProteinTech Group, Inc., Chicago, IL, USA), Beclin 1 (1:1,000; cat. no. 11306-1-AP; ProteinTech Group, Inc.), p62 (1:1,000; cat. no. 5114; Cell Signaling Technology, Inc.) and GAPDH (1:5,000; cat. no. 10494-1-AP; ProteinTech Group, Inc.). The membranes were washed three times ( $5 \mathrm{~min} /$ wash) with Tris-buffered saline containing $0.05 \%$ Tween- 20 . The membranes were then incubated with goat anti-rabbit immunoglobulin $\mathrm{G}(\mathrm{H}+\mathrm{L})$, horseradish peroxidase-conjugated secondary antibody (1:5,000; cat. no. SA00001-2; ProteinTech Group, Inc.) at $37^{\circ} \mathrm{C}$ for $1 \mathrm{~h}$. After washing of the membranes, enhanced chemiluminescence (ProteinTech Group, Inc.) was used to detect the signals. Quantity One v4.6.9 software (Bio-Rad Laboratories, Inc., Hercules, CA, USA) was used to scan the gray value of 
the blots, and GAPDH was used as an internal control. The relative expression levels were calculated by normalizing the target protein expression to the expression of the internal control.

Statistical analysis. Statistical analyses were performed using SPSS software (version 18.0; SPSS, Inc., Chicago, IL, USA). Data are expressed as the means \pm standard deviation. Statistical comparison between two groups was carried out with Student's t-test and one-way analysis of variance followed by a Dunnett's post hoc test was used to compare $>2$ groups. $\mathrm{P}<0.05$ was considered to indicate a statistically significant difference.

\section{Results}

ISL inhibits proliferation of MKN28 cells. A CCK8 assay was performed to detect the potential effects of ISL on the proliferation of gastric cancer cells. ISL treatment decreased the proliferation of MKN28 cells compared with in the NC group in a concentration-dependent manner (Fig. 1A). In addition, MKN28 cells were treated with ISL (20 $\mu \mathrm{M})$ for 0,24 , 48 and $72 \mathrm{~h}$. The OD values at 48 and $72 \mathrm{~h}$ were significantly different between the ISL and NC groups $(\mathrm{P}<0.05$; Fig. 1B), thus suggesting that ISL could effectively reduce MKN28 cell proliferation at $48 \mathrm{~h}$. In addition, the effects of ISL on GES-1 cell proliferation were investigated. The results indicated that ISL exerted little inhibitory effect on GES-1 cell proliferation (Fig. 1C). Therefore, subsequent experiments were performed only using MKN28 cells. In summary, ISL clearly inhibited MKN28 cell proliferation but exhibited little effect on GES-1 cell proliferation.

ISL inhibits migration and invasion of MKN28 cells. A Transwell assay was used to determine the effects of ISL on the migration and invasion of MKN28 cells (Fig. 2A). The Transwell migration assay revealed that the number of migrated cells in the ISL group was significantly decreased compared with in the NC group $(\mathrm{P}<0.05 ;$ Fig. $2 \mathrm{~B})$. In addition, the Transwell invasion assay demonstrated that the number of invaded cells was reduced in the ISL group compared with in the $\mathrm{NC}$ group (Fig. $2 \mathrm{C} ; \mathrm{P}<0.05$ ). These results indicated that ISL markedly reduced the invasion and migration of MKN28 cells.

ISL promotes apoptosis of MKN28 cells. The apoptosis of MKN28 cells was evaluated using the FITC Annexin V Apoptosis assay and flow cytometry, and apoptosis-associated proteins were detected by western blotting. The results demonstrated that treatment with ISL significantly increased cell apoptosis compared with in the NC group (Fig. 3A and B; $\mathrm{P}<0.05$ ). Western blotting (Fig. $3 \mathrm{C}$ ) indicated that the protein expression levels of Bcl-2 were reduced ( $\mathrm{P}<0.05$; Fig. 3D), whereas those of Bax were increased compared with in the NC group $(\mathrm{P}<0.05$; Fig. $3 \mathrm{E})$. Furthermore, the ratio of $\mathrm{Bax} / \mathrm{Bcl}-2$ was significantly increased following ISL treatment compared with in the NC group (Fig. 3F; $\mathrm{P}<0.05$ ), and active caspase-3 expression was increased following ISL administration (Fig. 3C and $\mathrm{G}$; $\mathrm{P}<0.05$ ). These results suggested that ISL may promote the apoptosis of MKN28 cells.
ISL triggers autophagy in MKN28 cells. Autophagy in MKN28 cells was detected by western blot analysis, which measured alterations in the protein expression levels of LC3I, LC3II, Beclin 1 and p62 (Fig. 4A). Compared with in the NC group, the ratio of LC3II/LC3I and the expression levels of Beclin 1 were clearly upregulated in response to ISL treatment, whereas p62 was downregulated ( $\mathrm{P}<0.05$; Fig. 4B-D). These results indicated that ISL may promote autophagy in MKN28 cells.

ISL downregulates the PI3K/AKT/mTOR signaling pathway in MKN28 cells. The effects of ISL on the expression levels of $\mathrm{PI} 3 \mathrm{~K} / \mathrm{AKT} / \mathrm{mTOR}$ signaling pathway-related proteins in MKN28 cells were investigated by western blot analysis, which was performed to analyze AKT, p-AKT, mTOR and p-mTOR protein expression (Fig. 5A). The protein expression levels of p-AKT were significantly decreased in ISL-treated MKN28 cells, with no significant differences in the total expression levels of AKT ( $\mathrm{P}<0.05$; Fig. 5B and C). Furthermore, p-mTOR was significantly decreased following ISL treatment, with no significant differences in the total mTOR levels $(\mathrm{P}<0.05$; Fig. 5D and E). These results suggested that the effects of ISL on MKN28 may be associated with downregulation of the $\mathrm{PI} 3 \mathrm{~K} / \mathrm{AKT} / \mathrm{mTOR}$ signaling pathway.

\section{Discussion}

The findings of the present study demonstrated that ISL inhibited the proliferation, migration and invasion of MKN28 cells. ISL also promoted apoptosis and autophagy in MKN28 cells. These effects may be associated with inhibition of the $\mathrm{PI} 3 \mathrm{~K} / \mathrm{AKT} / \mathrm{mTOR}$ signaling pathway.

ISL is a chalcone-type dietary flavonoid with relatively low toxicity; numerous studies have reported that ISL demonstrates potential antitumor activity. It has previously been reported that ISL inhibits cancer proliferation, including in prostate, oral and cervical cancer (19-21). In 2001, Ma et al (22) revealed that ISL may induce apoptosis of MGC-803 human gastric cancer cells through calcium- and Deltapsi (m)-dependent pathways. Lin et al (23) identified that ISL promotes MKN45 cell apoptosis, and enhances chemosensitivity in combination with 5-fluorouracil by targeting glucose-regulated protein 78. Previous studies have identified the marked antitumor activities of ISL, including migration inhibition, cell cycle arrest, apoptosis induction, initiation of oxidative stress and autophagy $(24,25)$; however, the effects of ISL on MKN28 cell proliferation, migration, invasion and autophagy have not been reported previously, to the best of our knowledge. The present study demonstrated that ISL may possess the ability to inhibit MKN28 cell proliferation, migration and invasion, and to induce apoptosis and trigger autophagy in MKN28 cells. These results are in line with previous findings in other cancers and imply that ISL may be a novel agent for gastric cancer treatment.

Cancer cells are characteristically aggressive, with the capabilities of immediate development and early metastasis. Extensive studies $(10,18,20,21,24)$ have demonstrated that agents that prevent tumorigenesis do so by disorganizing tumor initiation, proliferation, migration and invasion via cell death pathways, including apoptosis and autophagy. As important regulatory factors in the process of cell 
A

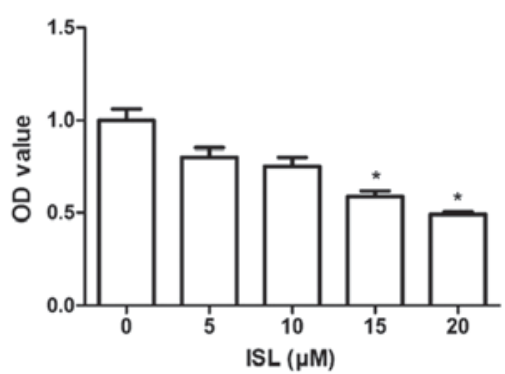

B

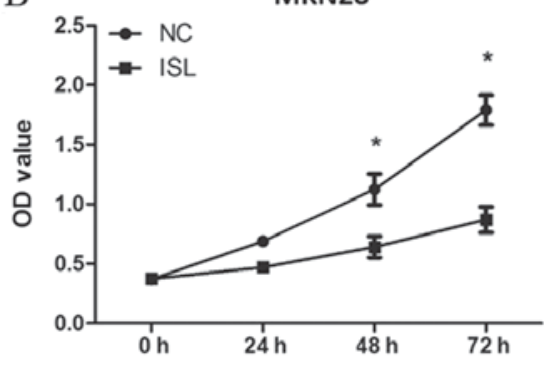

$\mathrm{C}$

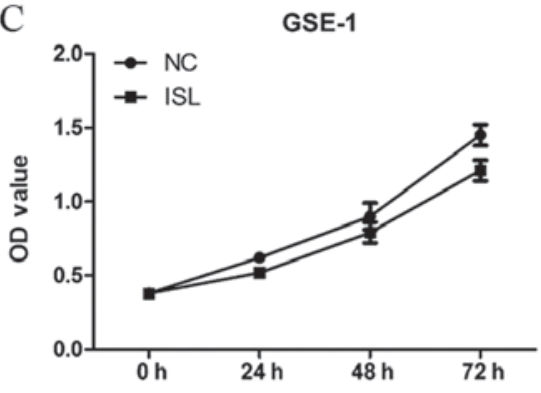

Figure 1. Antiproliferative activity of ISL on gastric cancer cells. MKN28 cells were treated with (A) various concentrations of ISL for 48 h, and (B) with $20 \mu \mathrm{M}$ ISL for various durations, and were evaluated by CCK8 assay. (C) Effects of ISL on GES-1 cell proliferation were evaluated by CCK8 assay. Results are presented as the means \pm standard deviation, $n=5$. ${ }^{*} \mathrm{P}<0.05$ vs. NC. CCK8, Cell Counting Kit-8; ISL, isoliquiritigenin; NC, negative control; OD, optical density.

A

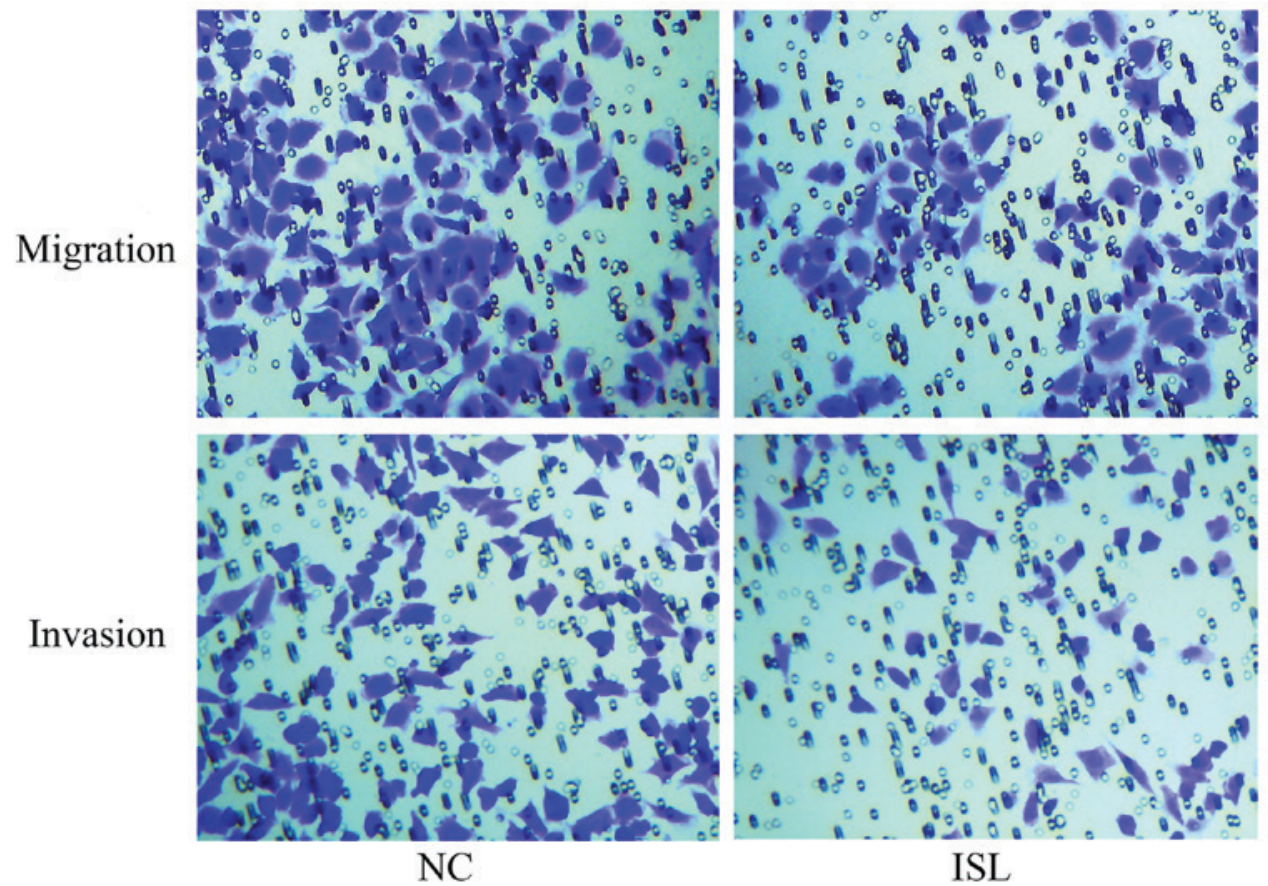

B

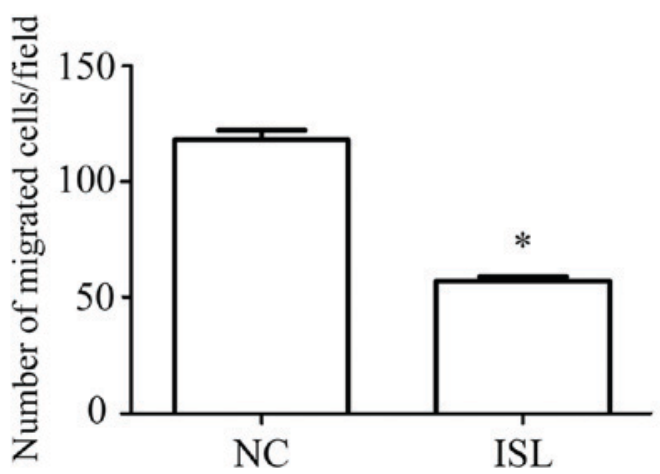

$\mathrm{C}$

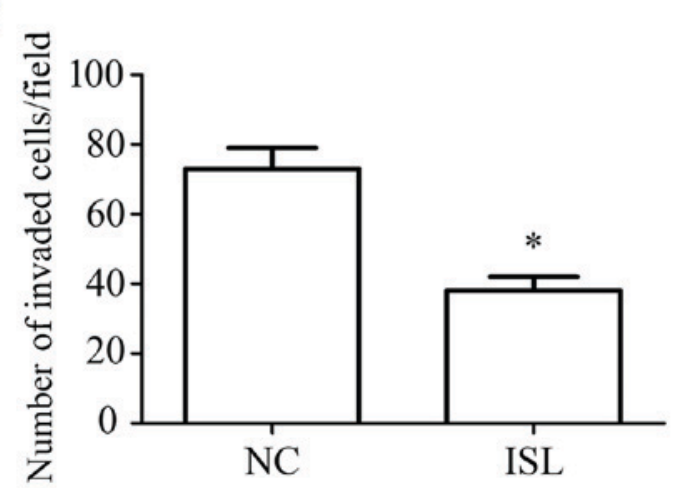

Figure 2. ISL suppresses migration and invasion of MKN28 cells. (A) Transwell assay demonstrated that ISL inhibited cell migration and invasion (magnification, x200). Quantification of (B) migration and (C) invasion. Results are presented as the means \pm standard deviation, $\mathrm{n}=5$. ${ }^{*} \mathrm{P}<0.05$ vs. NC. ISL, isoliquiritigenin; $\mathrm{NC}$, negative control.

apoptosis, Bcl-2 family members are divided into two groups: Anti-apoptotic proteins (Bcl-2) and pro-apoptotic proteins (Bax) $(26,27)$. Caspase-3 is downstream of the activator caspases. Therefore, activation of Bax and caspase-3 causes cells apoptosis (28). In the present study, western blot analysis indicated that Bax and caspase -3 expression levels were significantly increased, whereas Bcl-2 expression levels were significantly decreased. The results of the present study indicated that ISL may effectively induce MKN28 cell apoptosis, which is consistent with findings 
A

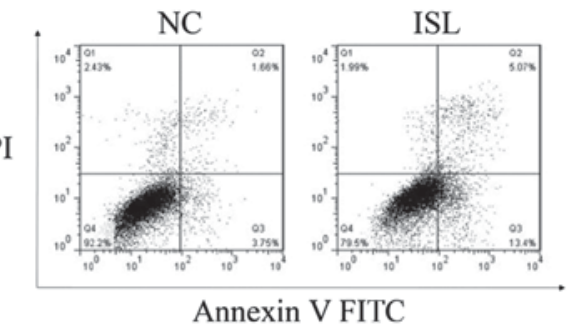

B

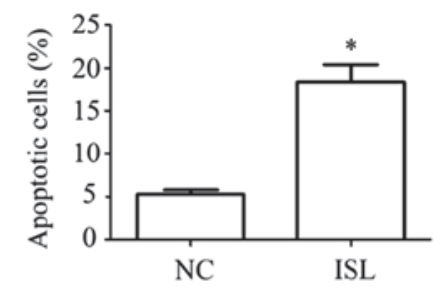

$\mathrm{C}_{\text {Bcl-2 }}$

Bax

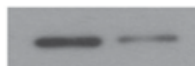

Active

caspase-3

GAPDH
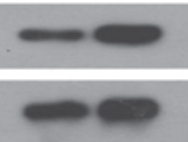
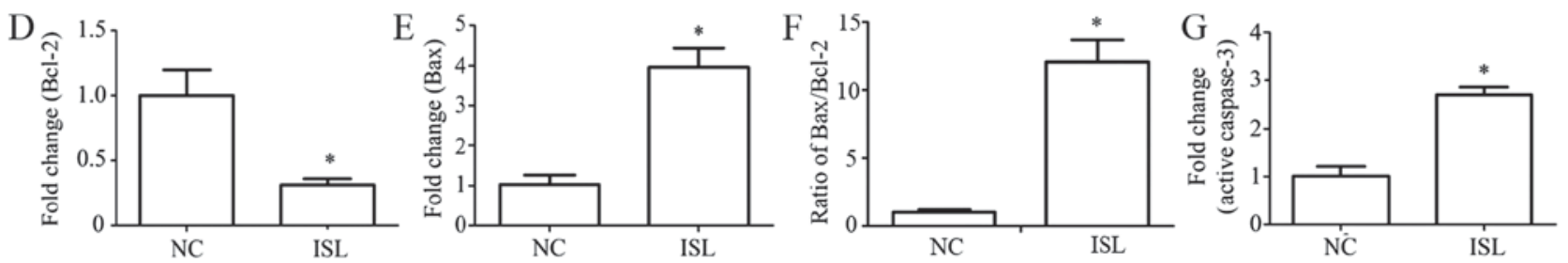

Figure 3. ISL promotes the apoptosis of MKN28 cells. (A) Cells were stained with Annexin V and PI, and apoptosis was measured using a flow cytometer (B) Quantification of apoptosis. (C) Bcl-2, Bax and active caspase-3 protein expression levels were detected by western blotting. GAPDH was used as a loading control. Statistical analysis of the protein expression levels of (D) Bcl-2 and (E) Bax following ISL treatment. (F) Bax/Bcl-2 ratio following ISL treatment (G) Statistical analysis of the protein expression levels of active caspase-3 following ISL treatment. Results are presented as the means \pm standard deviation, $\mathrm{n}=5$. "P<0.05 vs. NC. Bcl-2, B-cell lymphoma 2; Bax, Bcl-2-assoiated X protein; FITC, fluorescein isothiocyanate; ISL, isoliquiritigenin; NC, negative control; PI, propidium iodide.

A
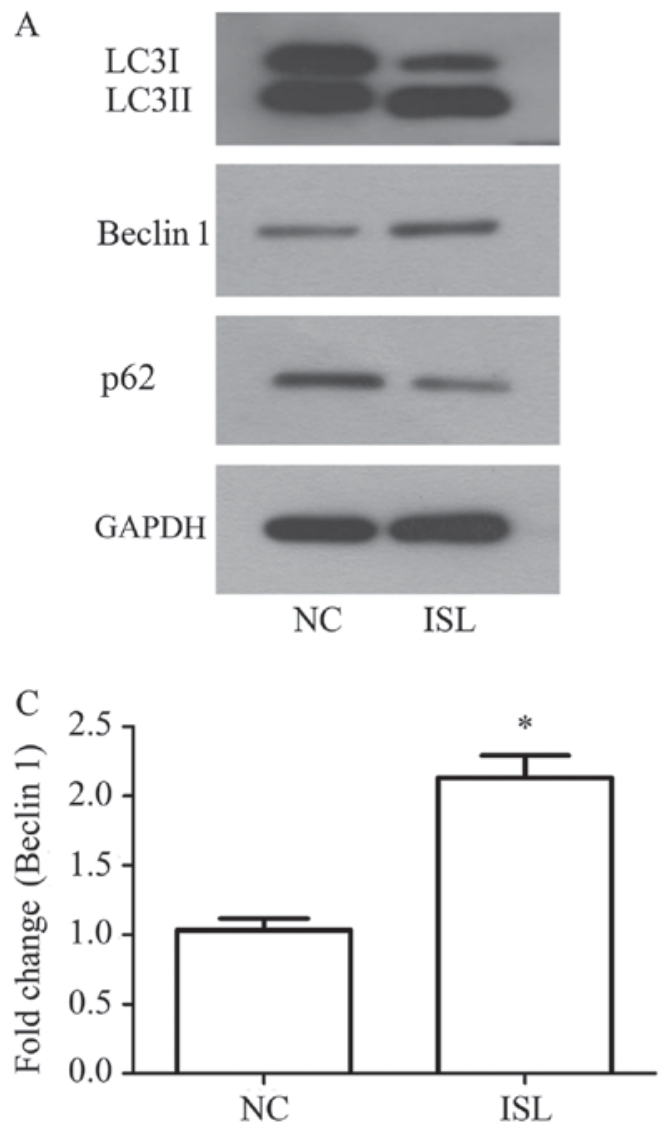

B
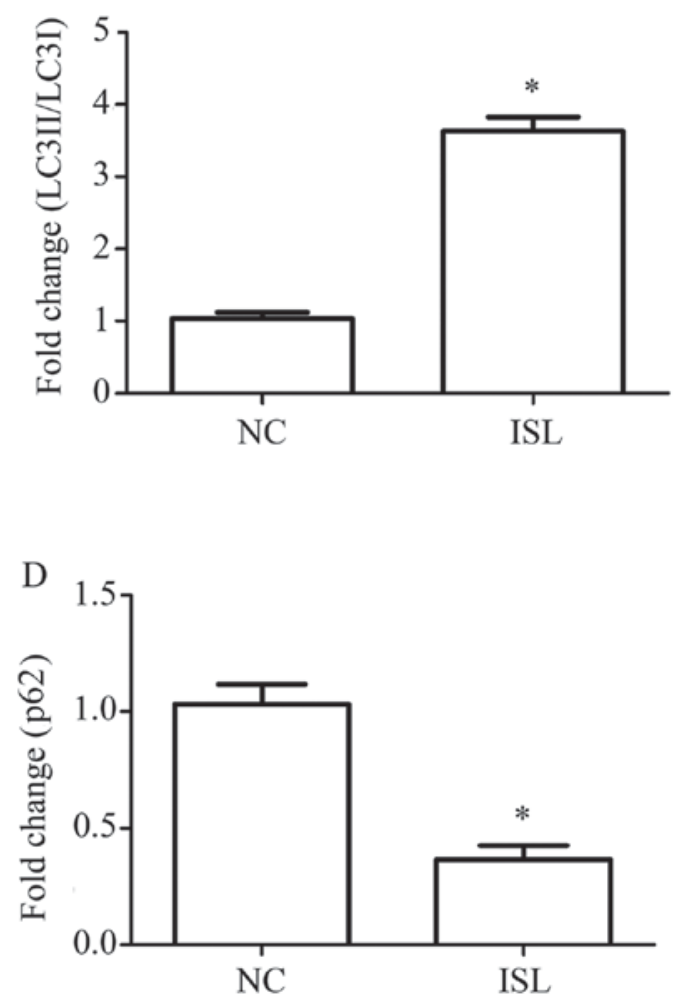

Figure 4. ISL triggers autophagy in MKN28 cells. (A) LC3II, LC3I, Beclin 1 and p62 protein expression levels were determined by western blotting. GAPDH was used as a loading control. Statistical analysis of the protein expression levels of (B) LC3II/LC3I, (C) Beclin 1 and (D) p62 following ISL treatment. Results are presented as the means \pm standard deviation, $n=5$. ${ }^{*} \mathrm{P}<0.05 \mathrm{vs}$. NC. ISL, isoliquiritigenin; $\mathrm{LC} 3$, microtubule-associated proteins $1 \mathrm{~A} / 1 \mathrm{~B}$ light chain $3 \mathrm{~B}$; $\mathrm{NC}$, negative control.

in T24 human bladder cancer cells (10). LC3 is a specific autophagy marker and LC3I is converted to LC3II during autophagy; therefore, the levels of LC3II/LC3I may indicate the occurrence of autophagy (29). The present study detected the ratio of LC3II/LC3I as an autophagy indicator. In general, autophagy is associated with decreased levels 
A

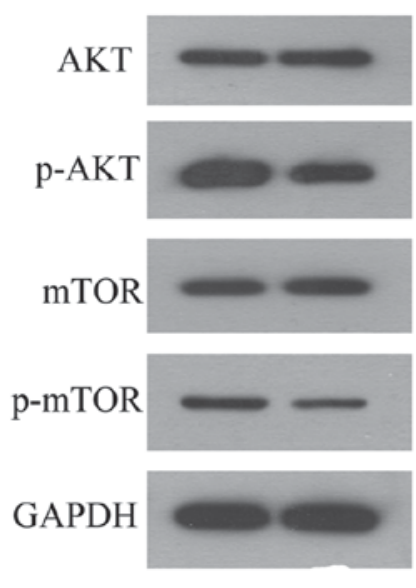

$\mathrm{B}$

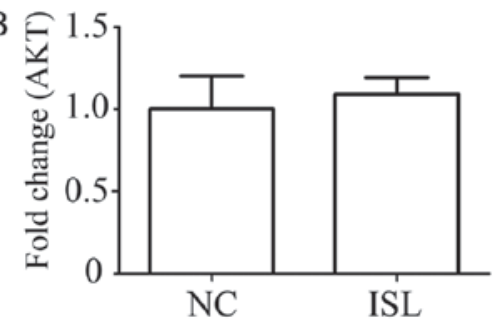

D

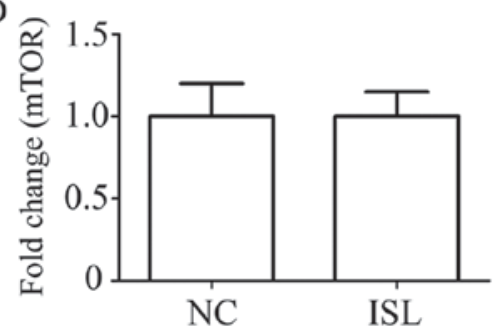

$\mathrm{C}$

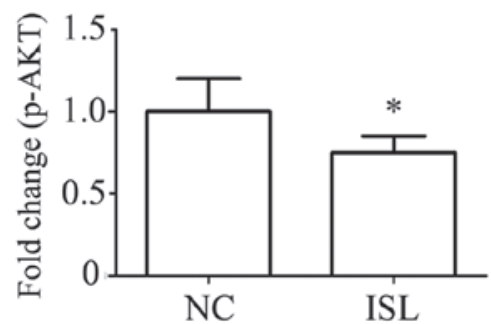

$\mathrm{E}$

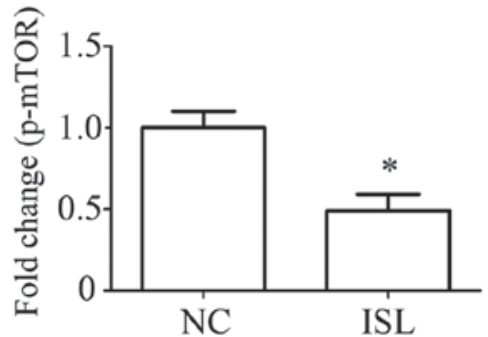

Figure 5. ISL downregulates the PI3K/AKT/mTOR signaling pathway in MKN28 cells. (A) AKT, p-AKT, mTOR and p-mTOR protein expression levels were determined by western blotting. GAPDH was used as a loading control. Statistical analysis of the protein expression levels of (B) AKT, (C) p-AKT, (D) mTOR and (E) p-mTOR following ISL treatment. Results are presented as the means \pm standard deviation, $\mathrm{n}=5$. * $\mathrm{P}<0.05$ vs. NC. AKT, protein kinase B; ISL, isoliquiritigenin; mTOR, mammalian target of rapamycin; NC, negative control; p-, phosphorylated.

of p62, suggesting that steady state levels of this protein reflect autophagy status (30). It is generally accepted that Beclin 1 is integral to the formation of autophagosomes in autophagy; therefore, the expression levels of the Beclin 1 were also investigated (31). The present study indicated that ISL significantly increased LC3II/LC3I expression levels, whereas p62 expression levels were markedly decreased in ISL-treated cells. Furthermore, the expression levels of Beclin 1 were markedly increased following ISL treatment.

Previous studies have concluded that ISL can affect cancer cell proliferation, migration, invasion, apoptosis and autophagy via various signaling pathway, including c-Jun $\mathrm{N}$-terminal kinase/activator protein-1, vascular endothelial growth factor (VEGF)/VEGF receptor 2 and PI3K/AKT $(19,32,33)$. The findings of the present study demonstrated that ISL may inhibit cancer via the PI3K/AKT/mTOR signaling pathway. The $\mathrm{PI} 3 \mathrm{~K} / \mathrm{AKT} / \mathrm{mTOR}$ signaling pathway serves an important role in cell proliferation and tumorigenesis (34-37). It is generally accepted that the PI3K/AKT/mTOR signaling pathway contributes to the proliferation of cancer cells. AKT participates in cell proliferation (38) and this pathway has also been reported to modulate cell apoptosis and growth (39), whereas mTOR regulates cell growth, metabolism and autophagy (40). Anticancer agents that target the PI3K/AKT/mTOR signaling pathway can induce apoptosis, autophagy and inhibit growth. For example, a marine sponge alkaloid derivative 4-chloro fascaplysin inhibits tumor growth by disrupting the PI3K/AKT/mTOR signaling cascade (41), and sinulariolide suppresses cell migration and invasion through the PI3K/AKT/mTOR signaling pathway in human bladder cancer cells (42).

ISL is a familiar dietary flavonoid that possesses antitumor properties. A previous study demonstrated that ISL serves as an inhibitor of the PI3K/AKT/mTOR signaling pathway in A375 melanoma cells (14). The present study investigated whether the PI3K/AKT/mTOR signaling pathway was involved in ISL-induced effects on MKN28 cells. It was identified that ISL indeed inhibited the PI3K/AKT/mTOR signaling pathway by downregulating the expression of $\mathrm{p}-\mathrm{AKT}$ and $\mathrm{p}$-mTOR. Therefore, it was hypothesized that, in vitro, ISL may inhibit proliferation, and induce autophagy and apoptosis, of MKN28 cells by regulating the $\mathrm{PI} 3 \mathrm{~K} / \mathrm{AKT} / \mathrm{mTOR}$ signaling pathway. However, the MKN-28 cell line has been reported to be a problematic/misidentified cell line, which is actually a MKN74 derivative (43). Fortunately, since they are both gastric cancer cell lines this has little effect on the results of the present study; however, caution must be exercised when analyzing the results and drawing conclusions.

In conclusion, the results of the present study indicated that ISL exerts antiproliferative, pro-apoptotic and autophagy effects on MKN28 cells, making it a promising candidate for gastric cancer treatment. Furthermore, suppression of the PI3K/AKT/mTOR signaling pathway and the subsequent expression of apoptosis and autophagy proteins may be a molecular mechanism underlying the antitumor effects of ISL. However, since this is a preliminary study, only one human gastric cancer cell line was used. The effects of ISL on more gastric cancer cell lines and animal models require further investigation in the near future. In addition, the effects of ISL on gastric cancer chemoprevention and the corresponding molecular mechanisms remain largely unknown and warrant further exploration.

\section{Acknowledgements}

Not applicable.

\section{Funding}

No funding was received.

\section{Availability of data and materials}

The data of the current study are available from the corresponding author on reasonable request. 


\section{Authors' contributions}

$\mathrm{XZ}$ and WS designed the present study. XZ and SW performed the experiments. CW helped to analyze the results and performed the statistical analysis. SW prepared the figures. XZ wrote the manuscript. WS and CW carefully revised the manuscript. All the authors have approved this manuscript.

\section{Ethics approval and consent to participate}

Not applicable.

\section{Patient consent for publication}

Not applicable.

\section{Competing interests}

The authors declare that they have no competing interests.

\section{References}

1. Ferlay J, Shin HR, Bray F, Forman D, Mathers C and Parkin DM Estimates of worldwide burden of cancer in 2008: GLOBOCAN 2008. Int J Cancer 127: 2893-2917, 2010.

2. Lin X, Zhao Y, Song WM and Zhang B: Molecular classification and prediction in gastric cancer. Comput Struct Biotechnol J 13: 448-458, 2015.

3. Torre LA, Bray F, Siegel RL, Ferlay J, Lortet-Tieulent J and Jemal A: Global cancer statistics, 2012. CA Cancer J Clin 65 : 87-108, 2015.

4. Liu W, Yang Q, Liu B and Zhu Z: Serum proteomics for gastric cancer. Clin Chim Acta 431: 179-184, 2014.

5. Asghari MH, Moloudizargari M, Ghobadi E, Fallah M and Abdollahi M: Melatonin as a multifunctional anti-cancer molecule: Implications in gastric cancer. Life Sci 185: 38-45, 2017.

6. Zheng R, Deng Q, Liu Y and Zhao P: Curcumin inhibits gastric carcinoma cell growth and induces apoptosis by suppressing the Wnt/beta-catenin signaling pathway. Med Sci Monit 23: 163-171, 2017.

7. Zheng YB, Xiao GC, Tong SL, Ding Y, Wang QS, Li SB and Hao ZN: Paeoniflorin inhibits human gastric carcinoma cell proliferation through up-regulation of microRNA-124 and suppression of PI3K/Akt and STAT3 signaling. World J Gastroenterol 21: 7197-7207,2015.

8. Shen H, Zhao S, Xu Z, Zhu L, Han Y and Ye J: Evodiamine inhibits proliferation and induces apoptosis in gastric cancer cells. Oncol Lett 10: 367-371, 2015.

9. Feng Yeh C, Wang KC, Chiang LC, Shieh DE, Yen MH and San Chang J: Water extract of licorice had anti-viral activity against human respiratory syncytial virus in human respiratory tract cell lines. J Ethnopharmacol 148: 466-473, 2013

10. Si L, Yang X, Yan X, Wang Y and Zheng Q: Isoliquiritigenin induces apoptosis of human bladder cancer T2 4 cells via a cyclin-dependent kinase-independent mechanism. Oncol Lett 14 241-249, 2017

11. Peng F, Du Q, Peng C, Wang N, Tang H, Xie X, Shen J and Chen J: A review: The pharmacology of isoliquiritigenin. Phytother Res 29: 969-977, 2015.

12. Zhao Z, Park SM, Guan L, Wu Y, Lee JR, Kim SC, Kim YW and Zhao R: Isoliquiritigenin attenuates oxidative hepatic damage induced by carbon tetrachloride with or without buthionine sulfoximine. Chem Biol Interact 225: 13-20, 2015.

13. Yadav VR, Prasad S, Sung B and Aggarwal BB: The role of chalcones in suppression of NF- $\mathrm{BB}$-mediated inflammation and cancer. Int Immunopharmacol 11: 295-309, 2011.

14. Chen XY, Li DF, Han JC, Wang B, Dong ZP, Yu LN, Pan ZH, Qu CJ, Chen Y, Sun SG and Zheng QS: Reprogramming induced by isoliquiritigenin diminishes melanoma cachexia through mTORC2-AKT-GSK3 $\beta$ signaling. Oncotarget 8: 34565-34575, 2017.
15. Wang Z, Wang N, Liu P, Chen Q, Situ H, Xie T, Zhang J, Peng C, Lin Y and Chen J: MicroRNA-25 regulates chemoresistance-associated autophagy in breast cancer cells, a process modulated by the natural autophagy inducer isoliquiritigenin Oncotarget 5: 7013-7026, 2014.

16. Zhao H, Yuan X, Li D, Chen H, Jiang J, Wang Z, Sun X and Zheng Q: Isoliquiritigen enhances the antitumour activity and decreases the genotoxic effect of cyclophosphamide. Molecules 18: 8786-8798, 2013.

17. Kim DH, Park JE, Chae IG, Park G, Lee S and Chun KS Isoliquiritigenin inhibits the proliferation of human renal carcinoma Caki cells through the ROS-mediated regulation of the Jak2/STAT3 pathway. Oncol Rep 38: 575-583, 2017.

18. Yoshida T, Horinaka M, Takara M, Tsuchihashi M, Mukai N, Wakada M and Sakai T: Combination of isoliquiritigenin and tumor necrosis factor-related apoptosis-inducing ligand induces apoptosis in colon cancer HT29 cells. Environ Health Prev Med 13: 281-287, 2008.

19. Kwon GT, Cho HJ, Chung WY, Park KK, Moon A and Park JH: Isoliquiritigenin inhibits migration and invasion of prostate cancer cells: Possible mediation by decreased JNK/AP-1 signaling. J Nutr Biochem 20: 663-676, 2009.

20. Hsia SM, Yu CC, Shih YH, Yuanchien Chen M, Wang TH, Huang YT and Shieh TM: Isoliquiritigenin as a cause of DNA damage and inhibitor of ataxia-telangiectasia mutated expression leading to $\mathrm{G} 2 / \mathrm{M}$ phase arrest and apoptosis in oral squamous cell carcinoma. Head Neck 38 (Suppl 1): E360-E371, 2016.

21. Hsu YL, Chia CC, Chen PJ, Huang SE, Huang SC and Kuo PL: Shallot and licorice constituent isoliquiritigenin arrests cell cycle progression and induces apoptosis through the induction of ATM/p53 and initiation of the mitochondrial system in human cervical carcinoma HeLa cells. Mol Nutr Food Res 53: 826-835, 2009.

22. Ma J, Fu NY, Pang DB, Wu WY and Xu AL: Apoptosis induced by isoliquiritigenin in human gastric cancer MGC-803 cells. Planta Med 67: 754-757, 2001.

23. Lin M, Wu D and Huang Y: Natural compounds ursolic acid and isoliquiritigenin target GRP78 to enhance human gastric cancer cell chemosensitivity by 5-fluorouracil. FASEB J 30: 1193-1194, 2016.

24. Wu CH, Chen HY, Wang CW, Shieh TM, Huang TC, Lin LC, Wang KL and Hsia SM: Isoliquiritigenin induces apoptosis and autophagy and inhibits endometrial cancer growth in mice. Oncotarget 7: 73432-73447, 2016.

25. Chen G, Zhu L, Liu Y, Zhou Q, Chen H and Yang J: Isoliquiritigenin, a flavonoid from licorice, plays a dual role in regulating gastrointestinal motility in vitro and in vivo. Phytother Res 23: 498-506, 2009.

26. Jergens A, Young J, Moore D, Wang C, Hostetter J, Augustine L, Allenspach K, Schmitz S and Mosher C: Bcl-2/caspase 3 mucosal imbalance favors $\mathrm{T}$ cell resistance to apoptosis in dogs with inflammatory bowel disease. Vet Immunol Immunopathol 158: 167-174, 2014.

27. Renault TT, Floros KV, Elkholi R, Corrigan KA, Kushnareva Y, Wieder SY, Lindtner C, Serasinghe MN, Asciolla JJ, Buettner C, et al: Mitochondrial shape governs BAX-induced membrane permeabilization and apoptosis. Mol Cell 57: 69-82, 2015.

28. Fang H, Wu Y, Guo J, Rong J, Ma L, Zhao Z, Zuo D and Peng S: $\mathrm{T}-2$ toxin induces apoptosis in differentiated murine embryonic stem cells through reactive oxygen species-mediated mitochondrial pathway. Apoptosis 17: 895-907, 2012.

29. Maiuri MC, Criollo A, Tasdemir E, Vicencio JM, Tajeddine N, Hickman JA, Geneste O and Kroemer G: BH3-only proteins and $\mathrm{BH} 3$ mimetics induce autophagy by competitively disrupting the interaction between Beclin 1 and Bcl-2/Bcl-X(L). Autophagy 3: 374-376, 2007.

30. Shvets E, Abada A, Weidberg H and Elazar Z: Dissecting the involvement of LC3B and GATE-16 in p62 recruitment into autophagosomes. Autophagy 7: 683-688, 2011.

31. Wei Y, An Z, Zou Z, Sumpter R, Su M, Zang X, Sinha S, Gaestel $M$ and Levine B: The stress-responsive kinases MAPKAPK2/MAPKAPK3 activate starvation-induced autophagy through Beclin 1 phosphorylation. elife 4: e05289, 2015.

32. Wang Z, Wang N, Han S, Wang D, Mo S, Yu L, Huang H, Tsui K, Shen J and Chen J: Dietary compound isoliquiritigenin inhibits breast cancer neoangiogenesis via VEGF/VEGFR-2 signaling pathway. PLoS One 8: e68566, 2013. 
33. Chen T, Deng S and Lin R: The inhibitory effect of Isoliquiritigenin on the proliferation of human arterial smooth muscle cell. BMC Pharmacol Toxicol 18: 57, 2017.

34. Chen G, Hu X, Zhang W, Xu N, Wang FQ, Jia J, Zhang WF, Sun ZJ and Zhao YF: Mammalian target of rapamycin regulates isoliquiritigenin-induced autophagic and apoptotic cell death in adenoid cystic carcinoma cells. Apoptosis 17: 90-101, 2012.

35. Wu S, Xue J, Yang Y, Zhu H, Chen F, Wang J, Lou G, Liu Y, Shi Y, Yu Y, et al: Isoliquiritigenin inhibits interferon- $\gamma$-inducible genes expression in hepatocytes through down-regulating activation of JAK1/STAT1, IRF3/MyD88, ERK/MAPK, JNK/MAPK and PI3K/Akt signaling pathways. Cell Physiol Biochem 37: 501-514, 2015.

36. Safdari Y, Khalili M, Ebrahimzadeh MA, Yazdani Y and Farajnia S: Natural inhibitors of PI3K/AKT signaling in breast cancer: emphasis on newly-discovered molecular mechanisms of action. Pharmacol Res 93: 1-10, 2015.

37. Wang ZG, Wang Y, Huang Y, Lu Q, Zheng L, Hu D, Feng WK, Liu YL, Ji KT, Zhang HY, et al: bFGF regulates autophagy and ubiquitinated protein accumulation induced by myocardial ischemia/reperfusion via the activation of the PI3K/Akt/mTOR pathway. Sci Rep 5: 9287, 2015.

38. Dillon RL, White DE and Muller WJ: The phosphatidyl inositol 3-kinase signaling network: Implications for human breast cancer. Oncogene 26: 1338-1345, 2007.
39. Chen L, Wang J, Wang B, Yang J, Gong Z, Zhao X, Zhang C and Du K: MiR-126 inhibits vascular endothelial cell apoptosis through targeting PI3K/Akt signaling. Ann Hematol 95: 365-374, 2016.

40. Arsham AM, Plas DR, Thompson CB and Simon MC: Phosphatidylinositol 3-kinase/Akt signaling is neither required for hypoxic stabilization of HIF-1 alpha nor sufficient for HIF-1-dependent target gene transcription. J Biol Chem 277: 15162-15170, 2002.

41. Sharma S, Guru SK, Manda S, Kumar A, Mintoo MJ, Prasad VD, Sharma PR, Mondhe DM, Bharate SB and Bhushan S: A marine sponge alkaloid derivative 4-chloro fascaplysin inhibits tumor growth and VEGF mediated angiogenesis by disrupting $\mathrm{PI} 3 \mathrm{~K} / \mathrm{Akt} / \mathrm{mTOR}$ signaling cascade. Chem Biol Interact 275: 47-60, 2017.

42. Cheng TC, Din ZH, Su JH, Wu YJ and Liu CI: sinulariolide suppresses cell migration and invasion by inhibiting matrix metalloproteinase-2/-9 and urokinase through the PI3K/AKT/mTOR signaling pathway in human bladder cancer cells. Mar Drugs 15: pii: E238, 2017.

43. Capes-Davis A, Theodosopoulos G, Atkin I, Drexler HG, Kohara A, MacLeod RA, Masters JR, Nakamura Y, Reid YA, Reddel RR and Freshney RI: Check your cultures! A list of cross-contaminated or misidentified cell lines. Int J Cancer 127: $1-8,2010$ 